\title{
Prevalencia de Maloclusiones en Niños y Adolescentes de 6 a 15 Años en Frutillar, Chile
}

\author{
Prevalence of Malocclusion in 6 to 15-year-old Children \\ and Adolescents in Frutillar, Chile
}

\author{
Daniela Burgos*
}

BURGOS, D. Prevalencia de maloclusiones en niños y adolescentes de 6 a 15 años en Frutillar, Chile. Int. J. Odontostomat., 8(1):13-19, 2014.

RESUMEN: Conocer la prevalencia de las maloclusiones es esencial para el desarrollo de estrategias preventivas, las cuales deben basarse en las necesidades de la población. El objetivo de esta investigación fue determinar la prevalencia de maloclusiones en niños y adolescentes de Frutillar, entre 6 a 15 años durante el año 2012, de acuerdo a sus características oclusales. En este estudio descriptivo, se realizó un examen clínico a 184 niños y adolescentes de Frutillar, seleccionados aleatoriamente a partir del total de escolares de establecimientos educacionales urbanos. En los cuales se evaluó la presencia de apiñamiento, espaciamiento, relación molar y canina, mordida cruzada posterior, mordida abierta lateral, escalón y resalte. Los datos fueron tabulados y expresados mediante el software LibreOffice. El 96,2\% de los estudiantes examinados presentó algún tipo de maloclusión, observándose con mayor frecuencia la discrepancia dentomaxilar negativa en un $67,4 \%$ de los casos. La prevalencia de maloclusiones encontrada, es mayor a la reportada por otros estudios. En Chile, existen pocos estudios publicados que revelen la real magnitud de las ADM, debido en parte a las distintas clasificaciones, diversos grupos etáreos evaluados y objetivo de cada estudio.

PALABRAS CLAVE: maloclusión, prevalencia, niños, adolescentes.

\section{INTRODUCCIÓN}

La prevalencia de las enfermedades orales más frecuentes en Chile, caries y gingivitis, han mostrado un descenso en las últimas dos décadas en menores de 20 años, gracias al desarrollo de estrategias preventivas (Cartes-Velásquez et al., 2010). Sin embargo, las maloclusiones, tercera alteración de mayor prevalencia, e impacto psicosocial (de Paula Júnior et al., 2009), no han sido incluidas en estas estrategias.

Realizar un tratamiento interceptivo, previene la progresión de las alteraciones (Tausche et al., 2004) y ayuda a disminuir el impacto negativo en el autoestima de los niños (Almeida et al., 2011). Por tanto, la prevención e intercepción oportuna, se considera conveniente en algunos tipos de maloclusiones, para disminuir sus consecuencias y aprovechar la capacidad de adaptación de los componentes tisulares del sistema estomatognático (Bustos et al., 2002).

Tanto en nuestro país (Gacitúa et al., 2000; Labranque et al., 2001; Soto et al., 2007), como a ni-

\footnotetext{
* Cirujano Dentista, Universidad Austral de Chile, Valdivia, Chile.
}

vel mundial (Onyeaso, 2004; Souza et al., 2008; Sidlauskas \& Lopatiene, 2009) se ha estudiado la prevalencia de maloclusiones, variando éstas de acuerdo al grupo étnico, rango etario o criterio diagnóstico utilizado. Richard \& Gantz (1972) revelaron que un 65\% a $72,6 \%$ de los niños chilenos entre 5 y 14 años presentaban algún tipo de maloclusión. Sin embargo, la mayoría de las investigaciones realizadas sobre éste tema, se han desarrollado en la Región Metropolitana, desconociendo la realidad en regiones.

Conocer la prevalencia de maloclusiones, es esencial para trazar un perfil de la comunidad en la cual se pretende instaurar medidas preventivas e interceptivas (Rosenfeld, 2008), como el programa de ortodoncia interceptiva que pretende instaurar el Módulo Dental, en Frutillar. El objetivo de este estudio fue determinar la prevalencia de maloclusiones, en niños escolares de Frutillar, entre 6 a 15 años, durante el año 2012, de acuerdo a sus características oclusales. 


\section{MATERIAL Y MÉTODO}

El presente estudio descriptivo transversal, fue llevado a cabo entre Abril y Julio del año 2012, donde la población objetivo fueron niños de 6 a 15 años, pertenecientes a establecimientos educacionales de la comuna de Frutillar, ubicada a 45 kilómetros de la capital regional, Puerto Montt, y a 980 kilómetros al sur de Santiago, que cuenta con una población total de 15.525 habitantes.

Previo al inicio de la investigación, se solicitó la aprobación del Departamento de Educación, Departamento de Salud de Frutillar, y el permiso de cada establecimiento educacional de dicha comuna.

Fueron incluidos estudiantes que al momento del examen, a realizarse a partir del 15 de Mayo del año 2012 hasta el 20 de Junio del mismo, tuvieran entre 6 y 15 años 11 meses cumplidos, que residieran en la ciudad de Frutillar, cuyos apoderados firmaran el consentimiento informado de participación en el estudio y los encargados de salud del establecimiento lo autorizaran. Se excluyeron estudiantes portadores de aparatología ortodóncica o con antecedentes de tratamiento ortodóncico previo, pacientes ASA III y IV, así como estudiantes de difícil manejo o que se negaran a participar de forma voluntaria en el estudio.

Se efectuó un muestreo aleatorio simple con el programa IBM SPSS Statistics 19 (Predictive analytics software) a partir del listado de alumnos matriculados que tengan entre 6 a 15 años 11 meses, de los distintos establecimientos educacionales urbanos de la comuna.

Se envió a cada uno de los apoderados de los alumnos seleccionados un consentimiento informado, el cual debió ser firmado por ellos, autorizando la participación de su pupilo en el estudio y devuelto por estos a su profesor jefe. Si alguno de los escolares seleccionados no presentó el consentimiento informado previo al examen, se seleccionó al escolar que se encontraba en la misma posición en una segunda tabla de aleatorización y en caso de no poder elegir nuevamente al individuo seleccionado, se escogió al que se encontraba en la misma posición en una tercera tabla de aleatorización realizada con el mismo Software. Finalmente, si los sujetos de estudio no presentaban el consentimiento, se solicitó la autorización a la encargada de salud del establecimiento educacional.
Posteriormente, se coordinaron las fechas y horarios en conjunto con los directores de los establecimientos educacionales, para realizar el examen clínico, actividad llevada a cabo en una sala especialmente habilitada para ello en el establecimiento educacional. Para éste, se empleó una lámpara portátil de espectro de luz blanco-azul (longitud de onda de alrededor de $495 \mathrm{~nm}$ ) y un set de instrumental de examen estéril. El paciente se encontraba recostado sobre una mesa, mientras el examinador se encontraba sentado detrás de la cabeza del sujeto. El examen clínico, fue realizado por una interna de Odontología de la Universidad Austral de Chile (UACh). Los datos obtenidos fueron registrados en la ficha clínica. Para cada edad, la cual evalúa los años cumplidos de los sujetos desde su nacimiento y cuya escala de clasificación fue de 6 a 15 , registrando las medidas obtenidas.

Las variables estudiadas fueron sexo, edad, tipo de dentición, presencia de compresión, relación canina y molar, resalte, escalón, presencia de mordida cruzada posterior, mordida abierta lateral, alteración intramaxilar y pérdida de espacio.

Se consideraron como datos perdidos, aquellos pacientes que no se encontraban en el establecimiento educacional durante los horarios acordados para el examen, y aquellos datos que estén incorrectamente ingresados en la ficha clínica o presenten omisiones. Las fichas correspondientes a los datos perdidos fueron incineradas.

El tamaño muestral fue calculado en base a un total de 2189 niños, entre 6 a 15 años de los establecimientos urbanos municipales y particulares subvencionados de Frutillar (DAEM, 2012), con una prevalencia esperada del $84,6 \%$ (Sidlauskas \& Lopatiene) y empleando un nivel de confianza del $95 \%$, con un error esperado del $5 \%$. El cálculo se realizó mediante la calculadora de tamaño muestral de Raosoft (Vovici, Systems Company), el cual arrojó un tamaño muestral de 184 individuos.

Se diseñó una guía para la correcta utilización de la ficha clínica, además de establecer criterios de medición para las variables y así facilitar la tabulación de datos, para su posterior análisis en base a las variables cuantitativas consignadas.

Los datos fueron ingresados y tabulados en un computador portátil marca Vaio serie FE, con sistema operativo Windows XP, mediante LibreOffice Windows, versión 3.5.5 (Productivity Suite). Para el procesamien- 
to de datos se utilizó la aplicación Calc, del mismo, que permite el desarrolló de tablas y gráficos para expresar los resultados obtenido relacionando por ejemplo, variables escalares como la edad en años y cuantitativas nominal como la distribución de una determinada maloclusión.

\section{RESULTADOS}

Se examinó un total de 225 pacientes, de los cuales fueron incluidos 184 (Fig. 1). Se excluyeron 38 registros debido a que estaban o habían sido tratados ortodóncicamente y también a estudiantes que no pertenecían a la comuna. Tres datos fueron eliminados por encontrarse incompletos. En la Figura 2, se detaIla la distribución de los pacientes, de acuerdo a la edad y sexo. De los pacientes examinados sólo 1 presentó dentición temporal; el $27,7 \%$ dentición mixta primera fase, el $25 \%$ dentición mixta segunda y el $46,7 \%$ presentó dentición permanente.

El $96,2 \%$ de los estudiantes examinados presentó algún tipo de maloclusión, de éstos el 44,6\% esta al límite o requería de tratamiento ortodóncico, de acuerdo al índice de necesidad de tratamiento ortodóncico (Tausche et al.).

Para el análisis de datos se agruparon los alumnos entre 6-9, 10-12 y 13-15 años, basado en la etapa de desarrollo de su dentición (Sidlauskas \& Lopatiene).

La maloclusión observada con mayor frecuencia fue la discrepancia dentomaxilar negativa en un $67,4 \%$ de los casos, donde un 5,4\% presentó apiñamiento en el maxilar, un $32,6 \%$ en la mandíbula y un $29,3 \%$ la presentó en ambos maxilares (Tabla I).

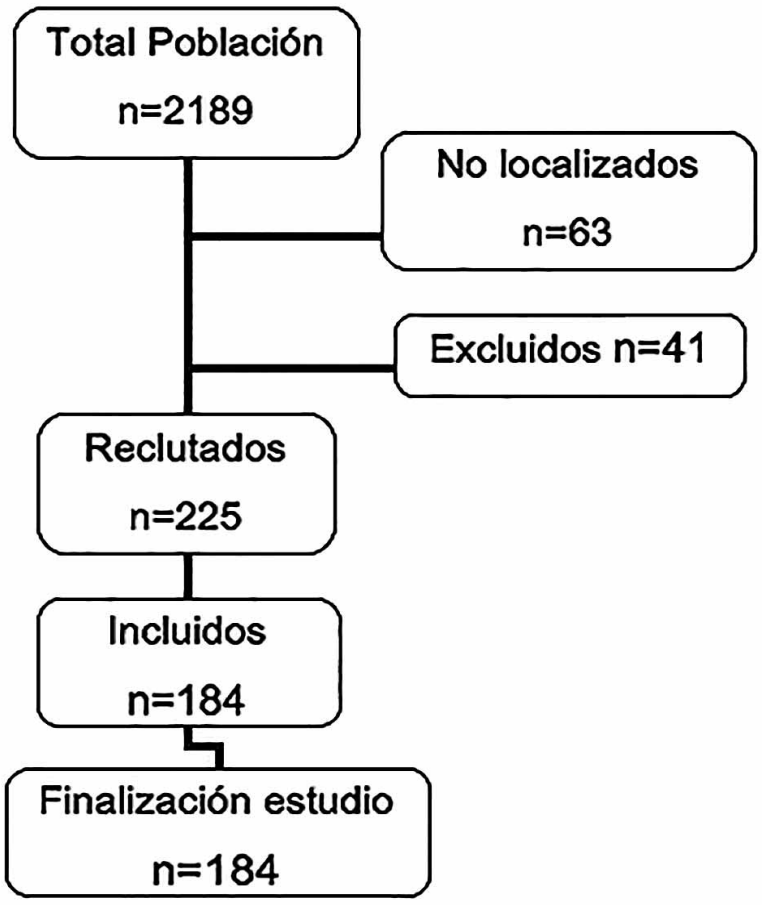

Fig. 1. Diagrama de Flujo para la selección de la muestra en la población de estudio.

La mordida abierta se observó en el 3,8\% de los sujetos evaluados, cuyo rango fluctúo entre los $-0,5$ a $-3 \mathrm{~mm}$. Mientras que la sobremordida con y sin contacto gingival se presentó en el $25 \%$, cuyas medidas iban desde los 4 hasta los $7 \mathrm{~mm}$ (Tabla II). Los resultados observados en cuanto a la medida del resalte, muestran que sólo un $2,7 \%$ presentó mordida invertida; y en un $22,8 \%$ de los sujetos se observó valores aumentados, cuyo valor máximo observado fue de $10 \mathrm{~mm}$ (Tabla III). En ambos casos el mayor número de alteraciones se observó en niños entre 10 a 12 años de edad.

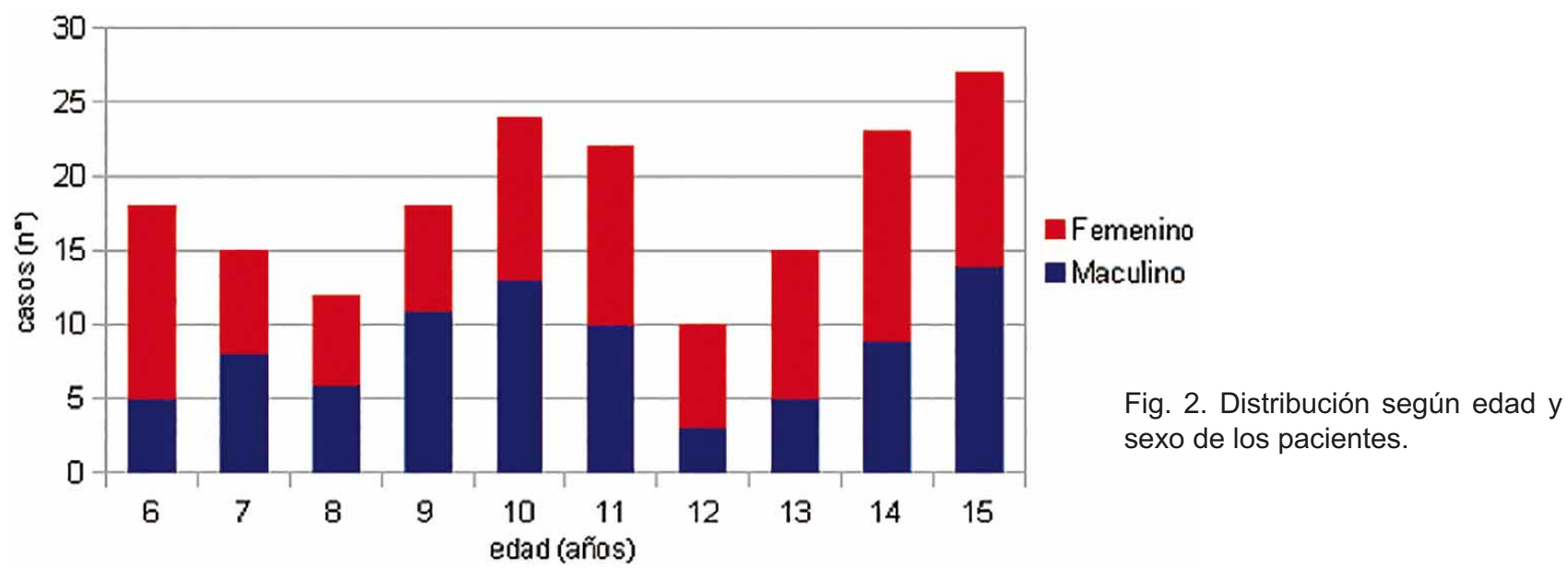


La mordida cruzada fue observada con mayor frecuencia en el lado derecho, además se presentó con mayor recurrencia en niños menores a 10 años (Tabla IV). No se observaron casos de mordida en tijera.

La relación canina observada con mayor frecuencia fue la normoclusión en el $47,3 \%$ de los casos, la distoclusión se vio en el $19 \%$ y la mesioclusión en el $13,6 \%$ de los sujetos evaluados (Tabla V).
La prevalencia de maloclusiones acorde a la Clasificación de Angle se presenta en la Tabla VI. La Clase I de Angle fue la más frecuente en el $51,1 \%$ de los sujetos, similar en ambos sexos; $24,5 \%$ presentó una Clase II y $21,7 \%$ de los casos mostró una Clase III de Angle. La relación molar del primer molar permanente no fue determinada en 5 escolares, debido a que había sido extraída o no se encontraba totalmente erupcionada.

Tabla I. Presencia de anomalía intramaxilar según tipo de discrepancia y maxilar.

\begin{tabular}{lcccc}
\hline \multirow{2}{*}{ Tipo de discrepancia } & \multicolumn{4}{c}{ Presencia discrepancia intramaxilar } \\
\cline { 2 - 5 } & Maxilar & Mandíbula & Ambos arcos & Total Resultado \\
\hline Apiñamiento & 10 & 60 & 54 & $\mathbf{1 2 4}$ \\
Espaciamiento & 11 & 4 & 8 & $\mathbf{2 3}$ \\
Total Resultado & & & & $\mathbf{1 4 7}$ \\
\hline
\end{tabular}

Tabla II. Distribución de la medida del escalón según grupo de edades.

\begin{tabular}{lcccccc}
\hline \multirow{2}{*}{ Grupo de edades } & \multicolumn{6}{c}{ Escalón en milímetros } \\
\cline { 2 - 7 } & $\mathbf{2} \mathbf{2 , 5}$ a $<\mathbf{4}$ & $\mathbf{2 4}$ & $\mathbf{< 0}$ & Igual a 0 En erupción & Total Resultado \\
\hline 6-9 años & 22 & 12 & 3 & 16 & 10 & 63 \\
10-12 años & 18 & 22 & 3 & 13 & & 56 \\
13-15 años & 28 & 12 & 1 & 24 & & 65 \\
Total Resultado & 68 & 46 & 7 & 53 & 10 & 184 \\
\hline
\end{tabular}

Tabla III. Distribución de la medida de resalte según grupo de edades.

\begin{tabular}{lcccccc}
\hline \multirow{2}{*}{ Grupo de edades } & \multicolumn{7}{c}{ Resalte en milímetros } \\
\cline { 2 - 7 } & $\mathbf{2 , 5} \mathbf{a}<\mathbf{4}$ & $\mathbf{2 4}$ & Igual a 0 & $<\mathbf{0}$ & En erupción & Total Re sultado \\
\hline 6-9 años & 27 & 14 & 11 & 1 & 10 & 63 \\
10-12 años & 27 & 19 & 8 & 2 & & 56 \\
13-15 años & 34 & 9 & 20 & 2 & & 65 \\
Total Resultado & 88 & 42 & 39 & 5 & 10 & 184 \\
\hline
\end{tabular}

Tabla IV. Distribución de acuerdo al tipo de mordida en la zona lateral en 184 pacientes, según el grupo de edades.

\begin{tabular}{lcccccc}
\hline \multirow{2}{*}{ Grupo de edades } & \multicolumn{5}{c}{ Tipo de mordida en la zona lateral } \\
\cline { 2 - 7 } & Normal & $\begin{array}{c}\text { Cruzada } \\
\text { Unilateral }\end{array}$ & $\begin{array}{c}\text { Cruzada } \\
\text { Bilateral }\end{array}$ & $\begin{array}{c}\text { Vis a vis } \\
\text { unilateral }\end{array}$ & $\begin{array}{c}\text { Vis a vis } \\
\text { bilateral }\end{array}$ & $\begin{array}{c}\text { Total } \\
\text { Resultado }\end{array}$ \\
\hline $6-9$ años & 44 & 5 & 8 & 5 & 1 & 63 \\
$10-12$ años & 43 & 2 & 2 & 4 & 5 & 56 \\
$13-15$ años & 55 & 3 & & 5 & 2 & 65 \\
Total Resultado & 142 & 10 & 10 & 14 & 8 & 184 \\
\hline
\end{tabular}

Tabla V. Distribución de acuerdo a la relación canina bilateral en 184 pacientes según grupo etario.

\begin{tabular}{lccccc}
\hline \multirow{2}{*}{ Grupo de edades } & \multicolumn{5}{c}{ Relación Canina } \\
\cline { 2 - 6 } & Normoclusión & Distoclusión & Mesioclusión & No aplicable & Total Resultado \\
\hline 6-9 años & 35 & 9 & 2 & 17 & 63 \\
$10-12$ años & 16 & 13 & 8 & 19 & 56 \\
13-15 años & 36 & 13 & 15 & 1 & 65 \\
Total Resultado & 87 & 35 & 25 & 37 & 184 \\
\hline
\end{tabular}


Tabla VI. Distribución de acuerdo a la relación molar bilateral y sexo en 184 pacientes

\begin{tabular}{|c|c|c|c|c|c|c|c|c|c|}
\hline \multirow{3}{*}{ Grupo de edades } & \multicolumn{9}{|c|}{ Relación Molar } \\
\hline & \multicolumn{2}{|c|}{ Normoclusión } & \multicolumn{2}{|c|}{ Distoclusión } & \multicolumn{2}{|c|}{ Mesioclusión } & \multicolumn{2}{|c|}{ No aplicable } & \multirow{2}{*}{ Total Resultado } \\
\hline & $\mathbf{M}$ & $\mathbf{F}$ & $\mathbf{M}$ & $\mathbf{F}$ & $\mathbf{M}$ & $\mathbf{F}$ & $\mathbf{M}$ & $\mathbf{F}$ & \\
\hline 6-9 años & 18 & 16 & 9 & 11 & 3 & 5 & & 1 & 63 \\
\hline 10-12 años & 13 & 11 & 9 & 11 & 4 & 7 & & 1 & 56 \\
\hline 13-15 años & 15 & 21 & 1 & 4 & 10 & 11 & 2 & 1 & 65 \\
\hline Total Resultado & 46 & 48 & 19 & 26 & 17 & 23 & 2 & 3 & 184 \\
\hline
\end{tabular}

$\mathrm{M}=$ Masculino; $\mathrm{F}=$ Femenino.

\section{DISCUSIÓN}

El presente estudio fue llevado a cabo con el fin de determinar la prevalencia de maloclusiones en niños y adolescentes de la comuna de Frutillar entre 6-9,10-12 y 13-15 años de acuerdo a sus características oclusales. El 96,2\% de los sujetos evaluados presentó algún tipo de desviación de la norma, siendo las anomalías intramaxilares más frecuentes que las intermaxilares. La anomalía más recurrente fue la discrepancia dentomaxilar negativa.

Se observó que el número de mujeres examinadas fue levemente superior a los hombres examinados. Mientras que, la distribución de la población en relación al tipo de dentición, y edades no fue homogénea. Además, la toma de mediciones en boca dificulta y crea un sesgo mayor para el análisis de los resultados.

La prevalencia de maloclusiones encontrada $(96,2 \%)$, es mayor a la reportada por otros estudios (Tabla VII). Sin embargo esto puede deberse a la diferencia en los criterios diagnósticos empleados y al rango etáreo evaluado.

Las relación molar I y II, según los criterios de
Angle, muestra resultados similares a los encontrados por otros autores (Tabla VII), sin embargo la Clase III, muestra una mayor prevalencia en comparación a otros resultados. Esto se puede deber al origen étnico de la población en estudio.

Encontramos un aumento en el resalte similar al reportada por Sidlauskas \& Lopatiene. Y valores en el escalón, mayores al mismo autor y a los reportados por Onyeaso y menores que los reportados por Keski-Nisula et al. (2003) y Martins \& Lima (2009).

Un importante porcentaje presentó anomalías intramaxilares $(79,9 \%)$; mayor al observado en otros estudios, como al $62,5 \%$ encontrado por Hererro (2003) en una población de entre 3-15 años de la isla de Juan Fernández y al 29,4\% observado por Gacitúa et al. en niños de 6 a 9 años en la comuna de Recoleta. Sin embargo, resulta difícil comparar poblaciones de distintas edades, y donde se emplearon criterios diagnósticos distintos.

El momento ideal en el cual comenzar el tratamiento de cada maloclusión, continúa siendo un tema controversial (Kluemper et al., 2000). El tratamiento

Tabla VII. Frecuencia de Anomalías Dentomaxilares en diversas poblaciones.

\begin{tabular}{|c|c|c|c|c|c|c|c|c|c|}
\hline Autor & País & $\begin{array}{l}\text { Edades } \\
\text { en } \\
\text { años }\end{array}$ & $\begin{array}{c}\text { Tamaño } \\
\text { de la } \\
\text { muestra }\end{array}$ & $\begin{array}{c}\text { Oclusión } \\
\text { normal } \\
\%\end{array}$ & $\begin{array}{c}\text { Clase I } \\
\text { de Angle } \\
\%\end{array}$ & $\begin{array}{c}\text { Clase II } \\
\text { de Angle } \\
\%\end{array}$ & $\begin{array}{c}\text { Clase III } \\
\text { de Angle } \\
\%\end{array}$ & $\begin{array}{c}\text { Escalón } \\
\text { Aumentado } \\
\%\end{array}$ & $\begin{array}{c}\text { Resal te } \\
\text { a umentado } \\
\%\end{array}$ \\
\hline $\begin{array}{l}\text { Iturriaga \& Whittle } \\
\text { (1990) }\end{array}$ & Chile & $5-20$ & 1000 & -- & 62,9 & 31,1 & 6 & -- & -- \\
\hline $\begin{array}{l}\text { Keski-Nisula et al. } \\
(2003)\end{array}$ & Finlandia & $4-7.8$ & 489 & -- & 46,1 & 52,4 & 1,5 & 33,0 & 38,8 \\
\hline Onyeaso (2004) & Nigeria & $12-17$ & 636 & 24.5 & 50,0 & 13,7 & 11,8 & 15,7 & 14,1 \\
\hline $\begin{array}{l}\text { Martins \& Lima } \\
\text { (2009) }\end{array}$ & Brazil & $10-12$ & 264 & 25.6 & 47,7 & 22,3 & 4,2 & 50,0 & 36,7 \\
\hline $\begin{array}{l}\text { Sidlauskas \& } \\
\text { Lopatiene (2009) }\end{array}$ & Lituania & $7-15$ & 1681 & -- & 68,42 & 27,66 & 2,79 & 21,09 & 14,46 \\
\hline
\end{tabular}


ortodóncico temprano es una opción que sugiere una serie de beneficios, como el uso del potencial de crecimiento de los huesos, prevención de la progresión de la maloclusión, disminución en la necesidad de extracciones y de cirugía ortognática, entre otros (Keski-Nisula et al.; Tausche et al.), es por esto, que el presente estudio consideró en la muestra niños de 6 años, edad en la cual comienza el período de dentición mixta temprana y con el fin de interceptar oportunamente las alteraciones para poder realizar la derivación necesaria en los casos que lo ameritó.

En Chile, existen pocos estudios publicados que revelen la real magnitud de las maloclusiones, debido en parte a las distintas clasificaciones, diversos grupos etáreos evaluados y objetivo de cada estudio (Labranque et al.). Por esto, es necesario emplear en futuros estudios, índices estandarizados como el índice de necesidad ortodóncico (IOTN) o el índice estético dental (DAl), que permitan homologar los resulta- dos a otras poblaciones, para así aumentar la validez externa del estudio. Además, se sugiere el uso de modelos de yeso en la realización de estudios similares que permita obtener resultados más fidedignos. También sería útil complementar los resultados obtenidos con telerradiografías, con el fin de determinar la relación de las alteraciones observadas con una maloclusión esqueletal subyacente.

\section{AGRADECIMIENTOS}

Al Departamento de Salud de la llustre Municipalidad de Frutillar y Dra. Sandra Álvarez, encargada Módulo Dental JUNAEB de Frutillar, por apoyar la realización de la investigación. A los Directores y Encargadas de Salud de los distintos establecimientos educacionales por facilitar y hacer más placentera el período de registro de datos.

BURGOS, D. Prevalence of malocclusion in 6 to 15-year-old children and adolescents in Frutillar, Chile. Int. J. Odontostomat., 8(1):5-11, 2014.

ABSTRACT: It is essential to determine the prevalence of malocclusions for the development of preventive strategies, which should be based on the needs of the population. The objective of this research was to determine the prevalence of malocclusion, in children and adolescents between 6 and 15 years of age in Frutillar, Chile in 2012, according to their occlusal characteristics. In this descriptive study, we performed clinical examinations in 184 schoolchildren in Frutillar. The children were randomly selected from the total number of students in urbans schools. We evaluated the presence of crowding, spacing, the relationship of the first upper and lower molars according to Angle's classification and canine relationship, posterior crossbite, lateral open bite, overbite and overjet. Data were tabulated and expressed by the LibreOffice software. Of the students tested $92 \%$ had some type of malocclusion, crowding being the most frequent and it was observed in $67.4 \%$ of cases. The prevalence of malocclusions found in this work, is greater than that reported in other studies. In Chile, there are few published studies that reveal the true magnitude of malocclusions in part due to the different classifications, age groups evaluated and the objective of each study.

KEY WORDS: malocclusion, prevalence, children, adolescents.

\section{REFERENCIAS BIBLIOGRÁFICAS}

Almeida, M. R.; Almeida, R. R.; Oltramari-Navarro, P. V.; Conti, A. C.; Navarro, R. L. \& Camacho, J. G. Early treatment of Class III malocclusion: 10-year clinical followup. J. Appl. Oral Sci., 19(4):431-9, 2011.

Bustos, A.; Mayorga, D. \& Espinoza, A. Prevalencia de Anomalías Dentomaxilares en Niños Escolares de 4 a 5 Años de Edad de la Comuna de La Calera. Rev. Dent. Chile, 93(1):3-8, 2002.

Cartes-Velásquez, R.; Araya, E. \& Valdés, C. Maloclusiones y su impacto psicosocial en estudiantes de un liceo intercultural. Int. J. Odontostomat., 4(1):65-70, 2010. de Paula Júnior, D. F.; Santos, N. C.; da Silva, E. T.; Nunes, M. F. \& Leles, C. R. Psychosocial Impact of Dental Esthetics on Quality of Life in Adolescents. Angle Orthod., 79(6):1188-93, 2009.

Gacitúa, G.; Mora, D.; Veloso, D. \& Espinoza, A. Prevalencia de anomalías dentomaxilares en Niños de 6 a 9 años en las escuelas municipales de la comuna de Recoleta. Rev. Dent. Chile, 91(3):27-30, 2000.

Herrero, C. Anomalías dentomaxilares, malos hábitos orales y alteraciones fonoarticulares en la población endogámica del archipiélago de Juan Fernández. Tesis de Pregrado, Santiago, Universidad de Chile, 2003. 
Iturriaga, R. \& Whittle, M. Analysis of 1000 cases of dentomaxillaty anomalies. Rev. Dent. Chile, 81(3):11623, 1990.

Keski-Nisula, K.; Lehto, R.; Lusa, V.; Keski-Nisula, L. \& Varrela, J. Occurrence of malocclusion and need of orthodontic treatment in early mixed dentition. Am. J. Orthod. Dentofacial. Orthop., 124(6): 631-8, 2003.

Kluemper, G. T.; Beeman, C. S. \& Hicks, E. P. Early orthodon.tic treatment: what are the imperatives? J. Am. Dent. Assoc., 131(5):613-20, 2000.

Labranque, R.; Contreras, P. \& Espinoza, A. Prevalence of Dentomaxilary Anomalies in a Schoolchildren Population of 5 to 6 Years of the Isla de Maipo Area. Rev. Dent. Chile, 92(1):7-12, 2001.

Martins, M. G. \& Lima, K. Prevalence of malocclusions in 10- to 12-year-old schoolchildren in Ceará, Brazil. Oral Health Prev. Dent., 7(3):217-23, 2009.

Onyeaso, C. O. Prevalence of malocclusion among adolescents in Ibadan, Nigeria. Am. J. Orthod. Dentofacial Orthop., 126(5):604-7, 2004.

Richard, C.; Gantz, R.; Cabrera, J. \& Ayala, J. Las anomalías dentomaxilares. Daño poblacional y realidad social. Rev. Dent. Chile, 62(1):147-9, 1972.

Rosenfeld, S. Estudio epidemiológico de maloclusiones en una población de 634 niños de entre 4 a 5 y 8 a 9 años de edad, de diversos colegios del área Metropolitana de Quito. Tesis de pregrado, Quito, Universidad de San Francisco, 2008.

Sidlauskas, A. \& Lopatiene, K. The prevalence of malocclusion among 7-15-year-old Lithuanian schoolchildren. Medicina (Kaunas), 45(2):147-52, 2009.

Soto, L.; Tapia, R.; Jara, G.; Rodríguez, G. \& Urbina, T. Diagnóstico nacional de salud bucal del adolescente de 12 años y evaluación del grado de cumplimiento de los objetivos sanitarios de salud bucal 2000-2010. Santiago, Facultad de Odontología, Universidad Mayor, 2007.

Souza, R. A.; Magnani, M. B. B. A.; Nouer, D. F.; Romano, F. L. \& Passos, M. R. Prevalence of malocclusion in a brazilian schoolchildren population and its relationship with early tooth loss. Braz. J. Oral Sci., 7(25):1566-70, 2008.

Tausche, E.; Luck, O. \& Harzer, W. Prevalence of malocclusions in the early mixed dentition and orthodontic treatment need. Eur. J. Orthod., 26(3):237-44, 2004.
Dirección para Correspondencia:

Daniela Burgos Leichtle

Cirujano Dentista

Universidad Austral de Chile

Valdivia

CHILE

Email: danii_burgos@hotmail.com

Recibido: 04-03-2013

Aceptado: 31-01-2014 